This item was submitted to Loughborough's Research Repository by the author.

Items in Figshare are protected by copyright, with all rights reserved, unless otherwise indicated.

\title{
Particulate clusters and permeability in porous media
}

PLEASE CITE THE PUBLISHED VERSION

http://dx.doi.org/10.1016/j.cherd.2011.11.019

\section{PUBLISHER}

(c) The Institution of Chemical Engineers. Published by Elsevier B.V.

\section{VERSION}

AM (Accepted Manuscript)

\section{LICENCE}

CC BY-NC-ND 4.0

\section{REPOSITORY RECORD}

Di Giovanni, B.A., Faiz M. Mahdi, Victor Starov, and R.G. Holdich. 2012. "Particulate Clusters and Permeability in Porous Media". figshare. https://hdl.handle.net/2134/10402. 
This item was submitted to Loughborough's Institutional Repository (https://dspace.lboro.ac.uk/) by the author and is made available under the following Creative Commons Licence conditions.

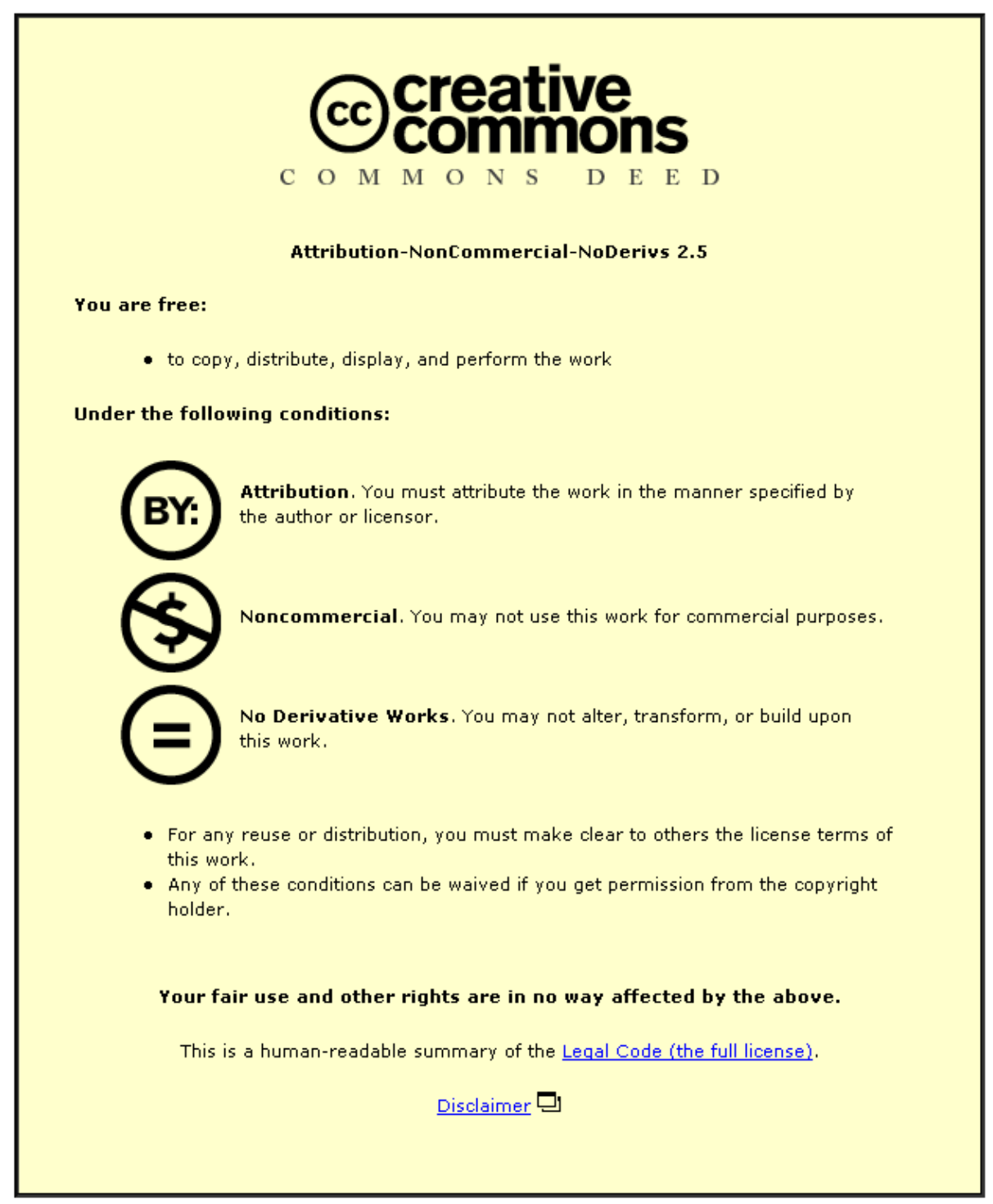

For the full text of this licence, please go to: http://creativecommons.org/licenses/by-nc-nd/2.5/ 


\title{
Particulate clusters and permeability in porous media
}

\author{
B. A. Di Giovanni, F. M. Mahdi, V. M. Starov, R. G. Holdich \\ Department of Chemical Engineering, Loughborough University, Loughborough, \\ Leicestershire, LE11 3TU, UK
}

\begin{abstract}
The permeability of particulate colloidal titanium dioxide, P25, was investigated during sedimentation, permeation and filtration when suspended in water at a consistent ionic strength similar to tap water. Happel's cell model of permeability was used to determine the apparent particle size during these processes, and compared with the size of particle clusters measured using laser diffraction under identical ionic conditions and varying degree of shear. The primary particle size of the P25 was determined to be $28 \mathrm{~nm}$, from consideration of the surface area and density of the particles, and the cluster size during permeation and filtration was close to $100 \mathrm{~nm}$. During sedimentation the cluster size was determined to be close to 10 microns, which is the same size obtained by laser diffraction when measuring under conditions of low shear. Using the above two sizes $(28 \mathrm{~nm}$ and $10 \mu \mathrm{m})$ as limits in Happel's permeability model it was possible to determine an 'operating envelope' of permeability that matched the experimentally measured values for the sedimentation, permeation and filtration processes.
\end{abstract}

Keywords: Permeation, cluster size, porous media, sedimentation, filtration.

Corresponding author:

Professor Richard Holdich, email: R.G.Holdich@Lboro.ac.uk

Department of Chemical Engineering, Loughborough University, Leics., LE11 3TU. 


\section{Introduction}

In any process where a fluid flows through a porous media, such as sedimentation, permeation and filtration, the permeability of the solids forming the porous medium governs the frictional loss. Hence, the observed rates of filtration, permeation and sedimentation are determined by that permeability, and process design and understanding would benefit from a method to reliably predict the permeability from a knowledge of the particle size distribution. This has been the desire of research workers over many years of study. Furthermore, the nature of the solids and how their particle size analysis is determined is also of great importance when dealing with finely sized particles, as they often form clusters containing many primary particles, and thereby influencing the predicted permeability. Clustering may be desirable in certain circumstances, such as to enhance sedimentation rate, but it will interfere with the particle size analysis, or characterisation, as the result will be dependent on the prevailing conditions during the analysis, which are likely to be very different to the conditions to be found in the process being designed.

There are a number of well-known permeability expressions [Carman, 1937, Brinkman, 1947] and most of them are derivatives of the Kozeny Carman equation [Xu and Yu, 2008]. However, these expressions contain an empirically determined parameter, e.g. the Kozeny 'constant' based on a model of the porous medium as a collection of tortuous channels. A better description of the microscopic flow field through a structure of interacting, but single and monosized spheres, has been presented by the Happel solution to the Navier-Stokes equation [Happel and Brenner, 1965]. Happel's cell model takes into consideration the drag forces acting on the individual spheres. Each sphere is assumed to be surrounded by an imaginary fluid 
region and the thickness of the outer fluid is chosen in order to have a voidage value of the cell (sphere + outer fluid) equal to the overall voidage in the medium.

The basic equation relating pressure drop $(\Delta P)$ and superficial velocity $\left(U_{0}\right)$ is Darcy's law, which for a single dimension is:

$\frac{\Delta P}{L}=-\frac{\mu}{k A} U_{O}$

where $k$ is the hydraulic permeability, $A$ is the cross sectional area to flow, $\mu$ is the coefficient of dynamic viscosity and $L$ is the bed height.

The best-known factors influencing the permeability of a porous medium are: particle size, particle size distribution (especially fines), solids concentration, particle shape and orientation. For fine particles, pre-treatment is usually used in order to facilitate the fluid flow: additives to modify the chemical and physical properties of the particles to promote interaction forming large entities that are easier to separate [Biggs, 2006].

In sedimentation the formation of clusters modifies the settling velocity [Michael and Bolger, 1962, Iritani et al., 2009] and in particular the sedimentation time. In cake filtration the presence of fines has always been a big problem, due to the occurrence of blinding and bleeding of the fines. The presence of strong clusters, stable enough to resist the shear conditions, overcomes these problems [Wu et al, 2003]. Voluminous and highly porous structured media may be formed by the clusters, which results in a much more permeable medium than the constituting fine particles [Gustafsson et al., 2003, Iritani, et al, 2009]. 
The clusters may be assumed to be spheres enabling experimental study and mathematical modelling [Happel and Brenner, 1965, Sunderland and Tan, 1970, Dixon et al., 1976, Font and Caballero, 2001]. Quantitative investigations based on the permeable status of the cluster has been done through the resolution of the Navier-Stokes equations for the flow exterior to the clusters; and Darcy's or Brinkman's equation for the internal flow [Neale et al. 1973, Matsumoto and Suganuma, 1977, Masliyah, 1987, Chellam and Wiesner, 1993, Vanni, 2000, Wu et al. 2002, Hsu 2009]. Radial permeability variation within the cluster has also been performed [Veerapaneni and Wiesner, 1996, Vanni, 2000, Wu et al. 2000, Li and Logan, 2001, Hsu et al., 2002, 2003, 2009, Yang, 2007] when considering flow within a large cluster (very large flocs of several hundred microns) in water treatment processes. The drag coefficient and settling factor are the main parameters used to explain the permeable status of the clusters [Chellam and Wiesner, 1993, Vanni, 2000, Li and Logan, 2001].

In the work reported here, cluster size and composition with applied shear is presented for dilute particle systems as measured by a standard particle size analysis technique for the finely divided inorganic solid particle, P25 titanium dioxide. The permeability of the porous medium constituted by the clustered solids is investigated during sedimentation, permeation and filtration; and the results compared with what is predicted from the particle size analysis data.

In the case of sedimentation, variations of the liquid $\mathrm{pH}$, ionic strength and temperature are well known to influence the separation process with the occurrence of sub-processes such as particle aggregation [Nabi, 2007, Iritani et al., 2009]. The 
permeability $(k)$ can be deduced from an overall force balance, considering the buoyed weight of the solids, the liquid drag and the solids stress gradient as showed in Equation (2):

$\frac{d P_{s}}{d z}=\left(\rho_{s}-\rho\right) g C-\frac{\mu}{k} U$

where $P_{s}$ is the solid pressure, $z$ is the height coordinate, $C$ is the solid concentration by volume fraction, $\rho$ is the liquid density, $\rho_{s}$ is the solid density, $g$ is the acceleration due to the gravity, and $U$ is the solid settling velocity. Under certain circumstances, solid stress and inertial terms may be neglected [Dixon et al., 1976, Holdich and Butt, 1996, Font and Caballero, 2001]. Hence, equation (2) can be rearranged for permeability using the experimentally measured settling velocity.

There are a number of published works investigating the influence of particle size and shape, particle size distribution, $\mathrm{pH}$ and nature of particle-particle interactions on filtration [Wakeman et al., 1991, Iritani et al., 1997, Wu et al., 2003]. In all cases, the particle size distribution within a cake is critically important for determining the permeability and, hence, the rate of filtration. However, it is the size (or size distribution) of the particle, or cluster of particles, that is important. Hence, it may be erroneous to measure the particle size distribution of solids under conditions that are not identical to those to be achieved within the filtration compact. Realistically, this is unlikely to be achieved and may cause concern over the relevancy (or otherwise) of particle size analysis to characterise material for filtration, or sedimentation, processes.

For the analysis of laboratory filtration tests two main approaches are adopted: constant pressure and constant rate filtrations. In the former case it is usual to plot a 
linear graph of filtration time-over-filtrate-volume $(t / \mathrm{V})$ against filtrate volume $(\mathrm{V})$, as described by the following equation:

$\frac{t}{V}=\frac{\mu c \alpha}{2 A^{2} \Delta P} V+\frac{\mu R_{m}}{A \Delta P}$

where $c$ is the dry mass of solids per unit filtrate volume (also known as the effective feed concentration in mass per unit volume units), $\alpha$ is the specific cake resistance and $R_{m}$ is the medium resistance. There are a number of methods and equations that can be used to determine the dry cake mass per unit volume of filtrate. If the cake concentration by volume fraction $(C)$ can be independently determined, possibly by material balance from a known starting concentration, then it is possible to use the equation:

$C=\frac{1}{\frac{(1-s) \cdot(1-C)}{s \rho}-\frac{\left(\rho_{S}\right.}{C}}$

where $s$ is the solid concentration of the slurry to be filtered as a mass fraction. If the cake filtration is carried out with a constant filtrate flow, due to an increase of the pressure across the filter during the formation of the cake, the fundamental equation (constant rate) is defined by Equation (5):

$\Delta P=\frac{\mu c \alpha V}{A^{2} t} V+\frac{\mu R_{m} V}{A t}$

In equation (5) the filtration pressure is a linear function of the filtrate volume. Both equations (3) and (5) assume incompressible filtration cake behaviour: i.e. pressure, volume and time are the only variables for a given filtration.

There is a simple relation between the specific cake resistance and permeability of the filter cake:

$k=\frac{1}{\rho_{s} C \alpha}$ 
A number of experimental techniques and analyses of the cake behaviour and structure have been published [Holdich et al., 1994, Tarleton, 2001] and these indicate that within a filter cake it is usual to have a reasonably uniform concentration throughout the cake so that the incompressible filtration equations may be applied, but that the solid concentration of the cake may differ between filtrations at different pressures. Hence, the occurrence of different values of cake concentration at different pressures does not invalidate the incompressible analysis of each individual filtration, so long as the appropriate value of concentration is used in that analysis. For the prediction of permeability, for comparison or use in equations (1), (2), (6) and, therefore, (3) and (5), the Happel and Brenner equation for permeability may be used:

$k=\frac{\left(2-3 C^{\frac{1}{3}}+3 C^{\frac{5}{3}}-2 C^{2}\right) X_{S V}^{2}}{12 C\left(3+2 C^{\frac{3}{2}}\right)}$

where $x_{s v}$ is the Sauter mean diameter of the particle distribution. For design purposes, equation (7) would be used after obtaining a particle size distribution, followed by the other equations for process modelling. In this work, equation (7) is used to rationalise the measured permeabilities, which are formed by the particle clusters.

\section{Materials and methods}

Commercial AEROXIDE ${ }^{\circledR}$ titanium dioxide P25 was purchased by Evonik Industries AG. This powder is produced through the so called Aerosil ${ }^{\circledR}$ process which is a hydrolysis of titanium tetrachloride $\left(\mathrm{TiCl}_{4}\right)$ at high temperature. Aggregates are formed of many spherical primary particles having a size close to the manufacturer specified value of $20 \mathrm{~nm}$ for P25, Figure 1 . Thus, it is correct to describe the primary particle size of this material as being approximately $20 \mathrm{~nm}$, but it is most likely that 
the particles have clustered together at the elevated temperature $\left(1000^{\circ} \mathrm{C}\right)$ used in the formation process, and the resulting cluster size will be greater than $20 \mathrm{~nm}$. The specific surface area was measured by a Micromeritics ASAP 2020 and found to be $53.19 \mathrm{~m}^{2} / \mathrm{g}$. The density was measured by a Micromeritics Multivolume Pycnometer 1305 and found to be $4030 \mathrm{~kg} / \mathrm{m}^{3}$. Treating the primary particles as spheres, these values of density and surface area provide a primary particle size of $28 \mathrm{~nm}$, assuming no internal particle surface area. Laser dynamic scattering (Horiba LA-920) was used to investigate cluster formation at different shear field values while the particles were dispersed in water containing electrolytes. In addition, a Particle Insight analyser (Micromeritics Instrument Corporation) was used to investigate the apparent shape of the clusters using image analysis.

Aqueous suspensions of P25 at different solid concentration were prepared. The solids were mixed with Reverse Osmosis (RO) water containing the electrolytes sodium nitrate (Fisher Scientific UK Ltd) and magnesium sulphate (Sigma-Aldrich Co.) as coagulants. The amount of both coagulants (1 mM each) was selected based on equalling the ionic strength of the local tap water. Initial tests of P25 in tap water showed good separation of the two phases, and clarity of the supernatant. Due to the possibility of variations in the tap water, a reproducible system based on RO water with added electrolytes was preferred. The natural $\mathrm{pH}$ value of the stored $\mathrm{RO}$ water, equal to 5.3, was maintained for all the tests.

Vacuum batch filtration tests were performed in a Plexiglass ${ }^{\circledR}$ cell with an inner diameter of $77.5 \mathrm{~mm}$. The filter medium employed had a nominal pore size of $2.7 \mu \mathrm{m}$ (WhatmanTM 542), and was fixed on top of a metal perforated plate. At the base of 
the cell, within the filtrate line, a pressure gauge (WIKA Instruments Ltd) and a pressure transducer (CPC Series Honeywell S\&C) were fixed in order to monitor the vacuum. The filtrate was pumped out using a peristaltic pump (Watson Marlow $101 \mathrm{U} / \mathrm{R}$ ) and collected in a vessel placed on an electronic balance (Ohaus TS4KV). The weight of the filtrate was recorded and a constant temperature was maintained for the filtration test. For the tests four different pump settings were used $(10 ; 40 ; 80$; and $99 \%$ of full-scale), which gave rise to four different shear stresses within the filter cake caused by increasing filtrate rate. The experimental equipment is illustrated in Figure 2, which shows schematically the filtration arrangement and the means by which permeation experiments were performed: by an overlying layer of supernatant sucked through the sediment.

\section{Results and Discussion}

The permeability of water through particulate solid structures, at different solid concentrations and final compactions, was investigated in the laboratory during:

sedimentation using equation 2, permeation using equation 1 and filtration using equation 6 . The intention being to compare the experimental permeability to the wellknown Happel's cell model, equation 7, taking into account possible particle clustering during the processes, and comparing the values with independently measured particle size data. Careful control of the experimental conditions was required for all the experiments and particular attention was taken to test the reproducibility of the experimental data at low shear conditions, primarily during sedimentation and particle size analysis. 


\subsection{Sensitivity of the data}

A preliminary investigation on the reproducibility of the data in sedimentation and particle size analysis (at low shear) was performed. This tested the control of the clustering process as well as the eventual possibility of modelling the kinetics of the clustering and the settling curves.

The reproducibility of sets of laser diffraction (LD) particle size measurements is shown in Figure 3: an initial reading at the lowest shear available in the LD device and after subjecting the sample to sonication for one minute. In total, seven replicate tests are shown in the figure: the initial cluster size (formed by adding 2 or 3 drops of suspension from the solids at a concentration of $1 \% \mathrm{v} / \mathrm{v}$ ) varied from 5.3 to 6.0 microns and the sonicated cluster size ranged from 1.6 to 1.9 microns. A high degree of repeatability of the data is evident between these tests.

For the sedimentation tests, the interface height between the supernatant and suspension, at different solid concentrations $(0.5 \% \mathrm{v} / \mathrm{v}, 0.7 \% \mathrm{v} / \mathrm{v}, 1.0 \% \mathrm{v} / \mathrm{v}$ and $1.5 \%$ v/v), was manually recorded. A set of initial tests at these concentrations, in different diameter vessels from $19 \mathrm{~mm}$ to $160 \mathrm{~mm}$, indicated that a vessel diameter of 160 $\mathrm{mm}$ provided a very similar settling curve to a $138 \mathrm{~mm}$ diameter vessel. Hence, the $160 \mathrm{~mm}$ diameter vessel was used and it was concluded that there is no effect on the settling due to vessel diameter. Before each sedimentation test, the vessel was filled with suspension maintained previously, for at least 24 hours, at constant temperature $\left(25+/-1^{\circ} \mathrm{C}\right)$ and periodically mixed. This ensured that the system had reached ionic equilibrium and avoided thermal gradients, both of which are fundamental for obtaining reproducible solid settling behaviour. It is known that finely divided particles can settle in a non-reproducible way [Shirato et al. 1970] making the 
design, or understanding, of sedimentation processes with these solids difficult. Hence, the careful control of the experimental conditions used in these tests. Following this procedure, the variation of interface height, at any instance in time, was found to be less than $5 \%$ over a series of settling tests. Thus, it was concluded that reproducible settling was achieved.

\subsection{Particle size analysis}

Ideally, in-situ cluster analysis is required. However, the relatively high concentration of dispersed phase and the consequent light scattering problems made this impossible. Cluster analysis using the Particle Insight analyser was attempted, but this requires samples to be taken and passed through an external flow cell to the system under investigation. A sample of the cluster images produced is illustrated in Figure 4. Many of the images are nearly spherical in nature, consistent with the expected cluster shape under mild shear, but the results are inconclusive and it was not possible to use the results to provide any meaningful insight in this system.

Further evidence of how easy the clusters can change is provided in Figure 5, where a series of tests at different pump speed through the circulation system of the LD system is reported with respect to analysis time. The circulation system installed in the LD equipment employs a pump allowing different pump speeds corresponding to different shear values. An analysis at different shear helps to illustrate both the kinetics of the clustering and the equilibrium cluster size.

Five different curves are shown in Figure 5: each curve corresponds to the kinetics of clustering at a specific value of shear, as given by the pump speed setting 
(arbitrary units). No attempt has been made to correlate the pump setting with energy input, but for the purpose of correlation with permeability data obtained from the sedimentation and filtration data the shear conditions can be described as simply 'low' or 'high' shear. Only at the lowest shear value did clustering occur to a significantly greater value than that initially formed: the Sauter mean diameter varied from an initial value of approximately $2 \mu \mathrm{m}$ up to about $9.4 \mu \mathrm{m}$. At higher shear, lower equilibrium diameters were obtained, as large clusters were prevented from forming. At the highest shear, the initial diameter was constant over the entire time investigated at a value of approximately 2.5 microns. The dispersion liquid in the LD equipment, to which the sample was added for the measurements, had the same ionic strength of the suspension from which it was taken, and used in all the tests reported later. A 'primary cluster' size value, equal to $0.1 \mu \mathrm{m}$, was obtained by the LD system by subjecting the sample to repeated intervals of sonication (up to a total of 20 minutes). Further sonication did not decrease the size any more. Thus, it is possible to deduce two main regions of cluster formation, starting with Sauter mean diameters, $0.1 \mu \mathrm{m}$ to $2 \mu \mathrm{m}$, which represents 'high strength' clusters (based on the primary particle size of $28 \mathrm{~nm}$ forming 'primary clusters' of diameter 0.1 to $2 \mu \mathrm{m}$ ). The second cluster size is between 2 and $9.4 \mu \mathrm{m}$, and these are 'low strength' clusters, where moderate shear can disrupt the 'secondary cluster' that is formed.

All the results are presented as Sauter mean diameter, the equivalent spherical diameter based on a particle that has the same specific surface area per unit volume as that of the entire distribution. This is the most appropriate diameter to use for permeability modelling, or correlation. 


\subsection{Fluid permeability on sedimentation, permeation and filtration}

The permeation of water through the structures formed during sedimentation, permeation and filtration processes was investigated. The structures were subjected to different shear stresses due to variation of the fluid flow. The size of the particulate clusters formed within the solid structures, and their variation with prevailing shear, has been deduced. Table 1 provides details of all the experimental conditions for each process.

In hindered sedimentation the fluid flows past the clusters without any external or added force. In order to use equation (2), for the purpose of determination of the permeability of the porous medium containing clusters, it is important to ensure that the solids stress gradient is negligible at the point in time when the calculation is performed. This was established by the use of liquid pressure measurements; a pressure transducer, placed at the bottom of the cell, was used to monitor the liquid pressure with time. It was noted, although no results are reported here, that at the start of the sedimentation the solids are fully suspended in the liquid and all the weight of the solids is supported by the liquid. Hence, the liquid is, in effect, a homogeneous liquid of density greater than that of the suspending water. During settlement the solids establish a solids network [Buscall and White, 1987] and a growing proportion of the weight of the solids will become supported by that network; i.e. the solids stress gradient will take some time to form and then increases with time. When all the solids have settled, the weight of the solids is no longer supported by the liquid drag term in equation (2); and all the weight is supported by the stress gradient. These liquid pressure tests provided readings of pressure that were within $5 \%$ of the expected pressure value using the above, fully liquid supported, rationale. 
Hence, it was appropriate to assume that the solids stress gradient was negligible and only the weight and drag terms in equation (2) need be considered.

In the case of filtration an initial test was performed using the solids suspended in RO water, with no electrolytes present. In this case the solids were dispersed well, showing no signs of cluster formation or sedimentation. Even under the gentlest of vacuum filtration conditions (i.e. the lowest setting on the pump used to suck water through the filter paper), it was not possible to form a filter cake. The finely dispersed solids were clearly sufficiently dispersed to pass through the pores of the filter medium and into the filtrate with no apparent loss of solids concentration, or filter cake formation. All the following reported filtrations were performed under the same ionic conditions used for the sedimentation and particle size analysis tests reported earlier. Under these conditions a filter cake formed readily. The permeability of the filter cake was assessed using equations (3), (5) and (6). In the case of permeation, the permeability was calculated simply using Darcy's law, equation (1).

Table 2 illustrates how the tests were performed, taking into account the natural tendency for the solids to settle and the response of the equipment used to perform the test work. In the latter case, the use of a peristaltic pump to suck the filtrate through the filter cake, or permeate compact. Hence, tests at low concentrations and low applied pressure (i.e. low pump speeds) showed significant sedimentation; so filtration tests were not possible under these conditions, only permeation tests after sedimentation was complete. Using the higher solids concentration, 2 and $3 \% \mathrm{v} / \mathrm{v}$, the cake readily formed and a constant pressure established: it was not possible to run under conditions of constant rate at these concentrations. For intermediate values of concentration and pressure both permeation as well as constant rate filtration tests were possible. 
Figure 6 shows the variation of solid concentration obtained from sedimentation, permeation and filtration tests. The solid compact concentration was determined by mass balance, from knowledge of the initial mass of solids used, and the final height of the compact formed during the sedimentation, filtration or permeation test. The uncertainty bars on the figure represent the minimum and maximum value that the concentration could possibly be, depending on the degree of precision of the measurement of the sediment, or cake height. This was measured to $+/-0.5 \mathrm{~mm}$, hence the concentrations reflect values calculated from the mass balance with an uncertainty of $+/-0.5 \mathrm{~mm}$ around the measured height after the test. It is assumed that the cake is of uniform concentration throughout its height. For the permeation tests, sedimentation preceded the permeation to form the compact to be tested, and then the overlying supernatant was sucked through the compact at a series of different pump settings and hence pressure drops. Despite the difference in pressure applied to the permeation tests the compact height, and therefore, solid concentration of the compact remained very stable; after an initial jump from the concentration formed during sedimentation of $3 \% \mathrm{v} / \mathrm{v}$ to the value obtained during permeation of between 8 to $9 \% \mathrm{v} / \mathrm{v}$.

By contrast, when filtering the solids from the start, i.e. in the absence of any sedimentation before filtration occurred, the filter cake concentration was a function of the applied filtration pressure. The concentrations of the vacuum formed filter cakes varying from 11 to $15 \% \mathrm{v} / \mathrm{v}$, roughly increasing with cake forming pressure, whereas the concentrations of the permeation cakes were roughly constant. Visual observation of the compacts supported these results: a noticeably wet cake was formed from the permeation tests and a stronger and much drier cake from filtration. 
In Figure 7, the experimentally determined permeability corresponding to the different solid concentrations for all three processes: sedimentation, permeation and filtration, is reported. The highest values of permeability are formed in sedimentation in which the clusters are uniformly suspended at low concentration in the porous medium. In permeation, the formation of a more compact solid bed is evident, with a decrease in permeability value, but with only very limited variation of solid concentration despite the different pressure used in the tests. It is logical that the variation in permeability is also limited for the permeation tests. The lowest permeability is found in filtration where maximum compaction of the cake occurs and there is a wide range of concentrations possible.

The permeability values obtained from the different tests have been converted to Sauter mean diameters, using equation (7) and plotted in Figure 8. In this conversion the Happel and Brenner cell model was rearranged for Sauter mean diameter and the measured values of both permeability and solid concentration were substituted into the equations. The data illustrated in Figure 8 covers two orders of magnitude for particle, or cluster, size and seven orders of magnitude for permeability. It suggests that the sedimentation tests had cluster size of close to 9.4 microns and for the highest shear filtration tests the particle size, determined from permeability, is slightly less than $100 \mathrm{~nm}$. This information conforms well to the data presented in Figures 1 and 5 . The inference is that in high shear filtration the filtrate flows rapidly through the filter cake and can easily penetrate, or disrupt, cluster formation. Hence, the correct particle size to use for permeability prediction in equation (7) would be between the primary particle size $(28 \mathrm{~nm})$ and the primary cluster size as determined by the LD tests using very high energy input $(100 \mathrm{~nm})$. At low shear, such as during 
sedimentation, the measured cluster size of 9.4 microns (see Figure 5) is appropriate. Hence, it is possible to use the particle/cluster sizes determined in Figure 5 in a predictive fashion for permeability as also illustrated in Figure 8, by the linear plots superimposed on this figure, described as follows. The sedimentation data was performed at starting concentrations between 0.5 and $1.5 \% \mathrm{v} / \mathrm{v}$, and gave rise to a sediment bed of $3 \% \mathrm{v} / \mathrm{v}$. Using these two values of starting solid concentration, together with the low shear cluster size of 9.4 microns, the starting points for the two lines in the top right hand corner of the graph are determined: two lines are required to cover the lowest and highest concentrations appropriate to sedimentation ( 0.5 and $3.0 \% \mathrm{v} / \mathrm{v})$. The ending point of the lines, in the bottom left hand corner of the graph, comes from the $28 \mathrm{~nm}$ value determined by from the surface area and density determinations. The explanation for using this value is that under high shear the fluid flow will be over the surface of the primary particles within the cake formed and not across any of the clusters. A solid concentration is also required, for use in equation (7), and in this case it is one provided by the measurement of cake height and material balance, in order to provide the final assumed uniform cake concentration. The two lines converge at this point, due to a single value of concentration used during the filtration, and the area between the two lines represents the operating envelope of expected permeability for the P25 material, over conditions of cluster formation and under conditions of different shear. The measured data, obtained over the different techniques, fits within the predicted operating envelope. If it is desired to obtain a relation between concentration and applied pressure, then this can be achieved from Figure 6 by fitting a power law equation through the data points: from sedimentation, the lowest pressure for permeation and the best fit between the filtration data. Such a plot, and its resulting equation, would enable the calculation of 
concentration for use in equation (7) for the purpose of permeability prediction. The correct 'cluster size' to use in the equation depends on the process itself: a low shear cluster size appears appropriate for sedimentation (9.4 microns), in permeation and filtration the cluster size appeared to be close to $100 \mathrm{~nm}$, the 'primary cluster' size measured by LD by the application of high energy input.

\section{Conclusions}

The prediction of permeability for the purpose of solid-liquid separation and contacting of colloidal suspensions is complicated by the variability of both concentration and apparent particle size due to cluster formation. Clustering can be beneficial, improving sedimentation rates and filtration permeability, but it is difficult to predict and control within designs. The work presented here investigated cluster formation using a conventional device for particle characterisation based on laser light diffraction. It was possible to correlate the resulting Sauter mean diameters arising from the analysis, at different shear conditions, with the apparent cluster (or particle) size observed during the operations of sedimentation, permeation and filtration of water through particle/cluster beds via the measured permeability and the predicted permeability based on Happel's cell model. For the purpose of design considerations, it is proposed that Happel's model can be used to provide the permeability of the clusters measured under low shear during particle characterisation. This, together with the suspension concentration, will provide the upper-limit of the expected permeability. Measurement of the primary particle size, was achieved by surface area measurement and density determination (and validated by SEM), and it was used to provide the absolute lower-limit of the expected permeability. The practical lower limit may come from the use of high 
energy input to disrupt the clusters during LD analysis. These values can be used to determine sedimentation and filtration rates during processing at, and between, these limits.

An unexpected result was the observation that during permeation the cluster size remained roughly constant, regardless of the rate of permeation applied. Hence, the compact was much more permeable than was obtained during filtration. This observation is consistent with reports of difficult filtrations being successfully achieved by allowing the colloidal material to settle first, before starting the filtration. Previously, this has been attributed to the minimisation of fine particles from entering the filter medium; it may be that cluster formation is another factor that assists in these filtrations.

The deduced cluster size during permeation and filtration was close to the value obtained by laser diffraction under conditions of high energy input (approximately $100 \mathrm{~nm})$, but significantly higher than the determined primary particle size (28 nm). Hence, although cluster formation is less relevant in permeation and filtration than sedimentation it clearly still plays an important role in determining permeability and, therefore, filtration rates.

In general, when characterising particles for use in sedimentation and filtration processes, it is to be recommended that the particles are measured in the same ionic medium to be used in practice and that a series of size analyses are performed at increasing energy, or shear, input. The primary particle size can be achieved from calculations using surface area and density measurement, and the results from the 
particle size measurements at different energy input can be compared with the primary particle size to determine if clustering is likely in the sedimentation and filtration processes.

\section{Acknowledgements}

BAD wishes to acknowledge the financial support of the UK, EPSRC. The work was undertaken as part of the DIAMOND project into Decommissioning, Immobilisation and Management of Nuclear wastes for Disposal. FMM is grateful to the Libyan Ministry of Higher Education for his scholarship. 


\section{References}

Biggs, S., 2006. Aggregates Structures and Solid-Liquid Separation Processes. KONA 24, 41-53.

Brinkman, H. C., 1947. A calculation of the viscous force exerted by a flowing liquid on dense swarm of particles. Appl. Sci. Res. A 1, 27-34.

Buscall, R. and White, L. R., 1987. The consolidation of concentrated suspensions. J. Chem. Soc., Faraday Trans. 83, 873-891.

Carman, P. C., 1937. Fluid flow through granular media. Trans. Inst. Chem. Eng. 15, 150-166.

Chellam, S. and Wiesner, M. R., 1993. Fluid mechanics and fractal aggregates. Water Res. 27 No 9, 1493-1496.

Dixon, D. C., Souter, P. and Buchanan, J. E., 1976. A study of inertial effects in sedimentation. Chem. Eng. Sci. 31, 737-740.

Font, R. and Cballero, J. A., 2001. Particulate character, inertial effects and diffusion effects in concentrated suspensions. Powder Technol. 120, 264-272. 
Gustafsson, J., Nordenswan, E. and Rosenholm, J. B., 2003. Consolidation behaviour in sedimentation of $\mathrm{TiO}_{2}$ suspensions in the presence of electrolytes. $\mathrm{J}$. Colloid Interface Sci. 258, 235-243.

Happel, J, Brenner, H, 1965. Low Reynolds Number Hydrodynamics. Prentice-Hall USA.

Holdich, R. G., Tarleton, E. S. and Willmer, S. A., 1994. Compressible cake filtration. Proc. IChemE Research Event, 1044-1046, IChemE, London.

Holdich, R. G., and Butt, G., 1996. Solid/liquid separation by sedimentation. Proc. Instn Mech. Engrs. 211 Part E, 43-52.

Hsu, J. P. and Hsieh, Y. H., 2002. Drag force on a floc in a flow field: two-layer model. Chem. Eng. Sci. 57, 2627-2633.

Hsu, J. P. and Hsieh, Y. H., 2003. Drag force on a porous, non-homogeneous spheroidal floc in a uniform flow field. J. Colloid Interface Sci. 259, 301-308.

Hsu, J. P., Chou, C. H., Tseng, S. and Wu, R. M., 2009. 3D simulations of hydrodynamic drag on a non-homogeneously structured permeable sphere and advective flow thereof. J. Colloid Interface Sci. 336, 850-856. 
Iritani, E., Toyoda, Y. and Murase, T., 1997. Effect of solution environment on deadend microfiltration characteristics of rutile suspensions. J. Chem. Eng. Jpn. 30 No. 4, 614-619.

Iritani, E., Hashimoto, T. and Katagiri, N., 2009. Gravity consolidation-sedimentation behaviours of concentrated $\mathrm{TiO}_{2}$ suspension. Chem. Eng. Sci. 64, 4414-4423.

Li, X. Y. and Logan, B. E., 2001. Permeability of fractal aggregates. Water Res. 35 No $14,3373-3380$.

Masliyah, J. H., Neale, G., Malysa, K. and Van De Ven, T. G. M., 1987. Creeping flow over a composite sphere: solid core with porous shell. Chem. Eng. Sci. 42 No. 2, 245-253.

Matsumoto, K. and Suganuma, A., 1977. Settling velocity of a permeable model floc. Chem. Eng. Sci. 32, 445-447.

Neale, G., Epstein, N. and Nader, W., 1973. Creeping flow relative to permeable spheres. Chem. Eng. Sci. 28, 1865-1874.

Michaels, A. S., and Bolger, J. C., 1962. Settling rates and sediment volumes of flocculated kaolin suspensions. Ind. Eng. Chem. Fundamen. 1 No. 1, 24-33.

Nabi, R. M., 2007. The influence of zeta potential on filtration performance. PhD. Thesis, The University of Leeds. 
Shirato, M., Kato, H., Kobayashi, K. and Sakazaki, H., 1970. Analysis of settling of thick slurries due to consolidation. J. Chem. Eng. Jpn. 3 No. 1, 98-104.

Sutherland, D. N. and Tan, C. T., 1970. Sedimentation of porous sphere. Chem. Eng. Sci. 25, 1948-1950.

Tarleton, E. S., 2001. The influence of sedimentation during downward cake filtration. Proc. Filtech Conference 20-27, Filtech Exhibitions, Dusserdolf.

Vanni, M., 2000. Creeping flow over spherical permeable aggregates. Chem. Eng. Sci. 55, 685-698.

Veerapaneni, S. and Wiesner, M. R., 1996. Hydrodynamics of fractal aggregates with radially varying permeability. J. Colloid Interface Sci. 177, 45-57.

Wakeman, R. J., Sabri, M. N. and Tarleton E. S., 1991. Factors affecting the formation and properties of wet compacts. Powder Technol. 65, 283-292.

Wu, R. M., Tsou, G. W. and Lee, D. J., 2000. Estimate of sludge floc permeability. Chem. Eng. J. 80, 37-42.

Wu, R. M., Tsou, G. W., Yen, P. S., Lee, D. J. and Peng, X. F., 2002. Tracking advective flow through floc interior. J. Chem. Eng. Jpn. 35 No. 6, 540-546. 
Wu, C. C., Wu, J. J. and Huang, R. Y., 2003. Effect of floc strength on sludge dewatering by vacuum filtration. Colloids Surf. A 221, 141-147.

Xu, P. and Yu, B., 2008.Developing a new form of permeability and Kozeny-Carman constant for homogeneous porous media by means of fractal geometry. Advances in Water Resources, 31, 74 - 81.

Yang, Z., Peng, X. F., Lee, D. J. and Su, A., 2007. Reynolds number-dependent permeability of wastewater sludge flocs. J CHIN INST CHEM ENG 38, 135-141.

\section{Nomenclature}

$A \quad$ is cross sectional area $(\mathrm{m})$

C is solid concentration by volume fraction (-)

c is dry mass of solids per unit filtrate volume $\left(\mathrm{kg} / \mathrm{m}^{3}\right)$

$g \quad$ is acceleration due to the gravity $\left(\mathrm{m} / \mathrm{s}^{2}\right)$

$k \quad$ is hydraulic permeability $\left(\mathrm{m}^{2}\right)$

$L \quad$ is bed height $(\mathrm{m})$

$P_{s} \quad$ is solid pressure $(\mathrm{Pa})$

$R_{m} \quad$ is medium resistance $\left(\mathrm{m}^{-1}\right)$

$\mathrm{S} \quad$ is solid concentration of the slurry by mass fraction (-)

$\mathrm{t} \quad$ is time (s) 
$U_{0} \quad$ is superficial velocity $(\mathrm{m} / \mathrm{s})$

$U \quad$ is solid settling velocity $(\mathrm{m} / \mathrm{s})$

$V \quad$ is volume of filtrate $\left(\mathrm{m}^{3}\right)$

$x_{S V} \quad$ is Sauter mean diameter $(\mathrm{m})$

$\mathrm{z} \quad$ is height coordinate $(\mathrm{m})$

\section{Greek letters}

$\alpha \quad$ is specific cake resistance $(\mathrm{m} / \mathrm{kg})$

$\Delta P \quad$ is pressure drop $(\mathrm{Pa})$

$\mu \quad$ is liquid viscosity ( $\mathrm{Pa} \mathrm{s}$ )

$\rho \quad$ is liquid density $\left(\mathrm{kg} / \mathrm{m}^{3}\right)$

$\rho_{s} \quad$ is solid density $\left(\mathrm{kg} / \mathrm{m}^{3}\right)$ 


\section{List of Tables}

Table 1 Experimental conditions used in sedimentation, permeation and filtration tests

Table 2 Test conditions in filter cell for the different initial solid concentration suspensions and various pump suction settings where CR is Constant Rate and $\mathrm{P}$ is constant Pressure filtration 


\section{List of Figures}

Figure 1 Scanning electron microscope image of P25 titanium dioxide

Figure 2 Experimental setup in filtration $(A)$, permeation $(A \& B)$ and sedimentation $(B)$ tests

Figure 3 Sauter mean diameter of P25 clusters initially and after 1 minute of sonication at the lowest shear field under LD for 7 repeated experiments

Figure 4 Cluster shapes determined by off-line image analysis: a sample of 48 of the images used

Figure $5 \quad$ Variation of the Sauter Mean Diameter over time for five different values of shear fields created by increasing pump speed from 1 (lowest value) to 5 (highest) during the LD analysis

Figure 6 Dependence of solid cake concentrations on pressure in filtration, permeation and sedimentation processes

Figure 7 Deduced permeability with solid concentration in filtration, permeation and sedimentation tests

Figure 8 Sauter mean diameter and corresponding permeability deduced from experimental readings and the predicted operating envelope based on particle characterisation 
Table 1 Experimental conditions used in sedimentation, permeation and filtration tests

\begin{tabular}{|c|c|c|c|}
\hline Parameter & Sedimentation & Permeation & Filtration \\
\hline $\begin{array}{c}\text { Initial solid } \\
\text { concentration } \\
(\% \mathrm{v} / \mathrm{v})\end{array}$ & $\begin{array}{c}0.3,0.5,0.7,0.9, \\
1.0,1.5\end{array}$ & $0.7,1.0,1.5$ & $0.7,1.0,1.5,2.0$, \\
\hline $\begin{array}{c}\text { Pump speed (\%) } \\
\text { Duration separation } \\
\text { (minutes) }\end{array}$ & 210 to days & $\begin{array}{c}\text { Min: } 30 \\
\text { Max: } 90\end{array}$ & $\begin{array}{c}\text { Min: } 15 \\
\text { Max: } 720\end{array}$ \\
\hline
\end{tabular}


Table 2 Test conditions in filter cell for the different initial solid concentration suspensions and various pump suction settings where CR is Constant Rate and $\mathrm{P}$ is constant Pressure filtration

\begin{tabular}{|c|c|c|c|c|}
\hline Pump speed: & $\mathbf{1 0}$ & $\mathbf{4 0}$ & $\mathbf{8 0}$ & $\mathbf{9 9}$ \\
\hline $\mathbf{0 . 7} \% \mathbf{v l v}$ & permeation & permeation & $\begin{array}{c}\text { permeation \& } \\
\text { CR filtration }\end{array}$ & $\begin{array}{c}\text { permeation \& } \\
\text { CR filtration }\end{array}$ \\
\hline $\mathbf{1 . 0} \% \mathbf{v l v}$ & permeation & $\begin{array}{c}\text { permeation \& } \\
\text { CR filtration }\end{array}$ & $\begin{array}{c}\text { permeation \& } \\
\text { CR filtration }\end{array}$ & $\begin{array}{c}\text { permeation \& } \\
\text { CR filtration }\end{array}$ \\
\hline $\mathbf{1 . 5} \% \mathbf{v l v}$ & permeation & $\begin{array}{c}\text { permeation \& } \\
\text { CR filtration }\end{array}$ & $\begin{array}{c}\text { permeation \& } \\
\text { CR filtration }\end{array}$ & $\begin{array}{c}\text { permeation \& } \\
\text { CR filtration }\end{array}$ \\
\hline $\mathbf{2 . 0} \% \mathbf{v l v}$ & P filtration & P filtration & P filtration & P filtration \\
\hline $\mathbf{3 . 0} \% \mathbf{v l v}$ & - & P filtration & P filtration & P filtration \\
\hline
\end{tabular}




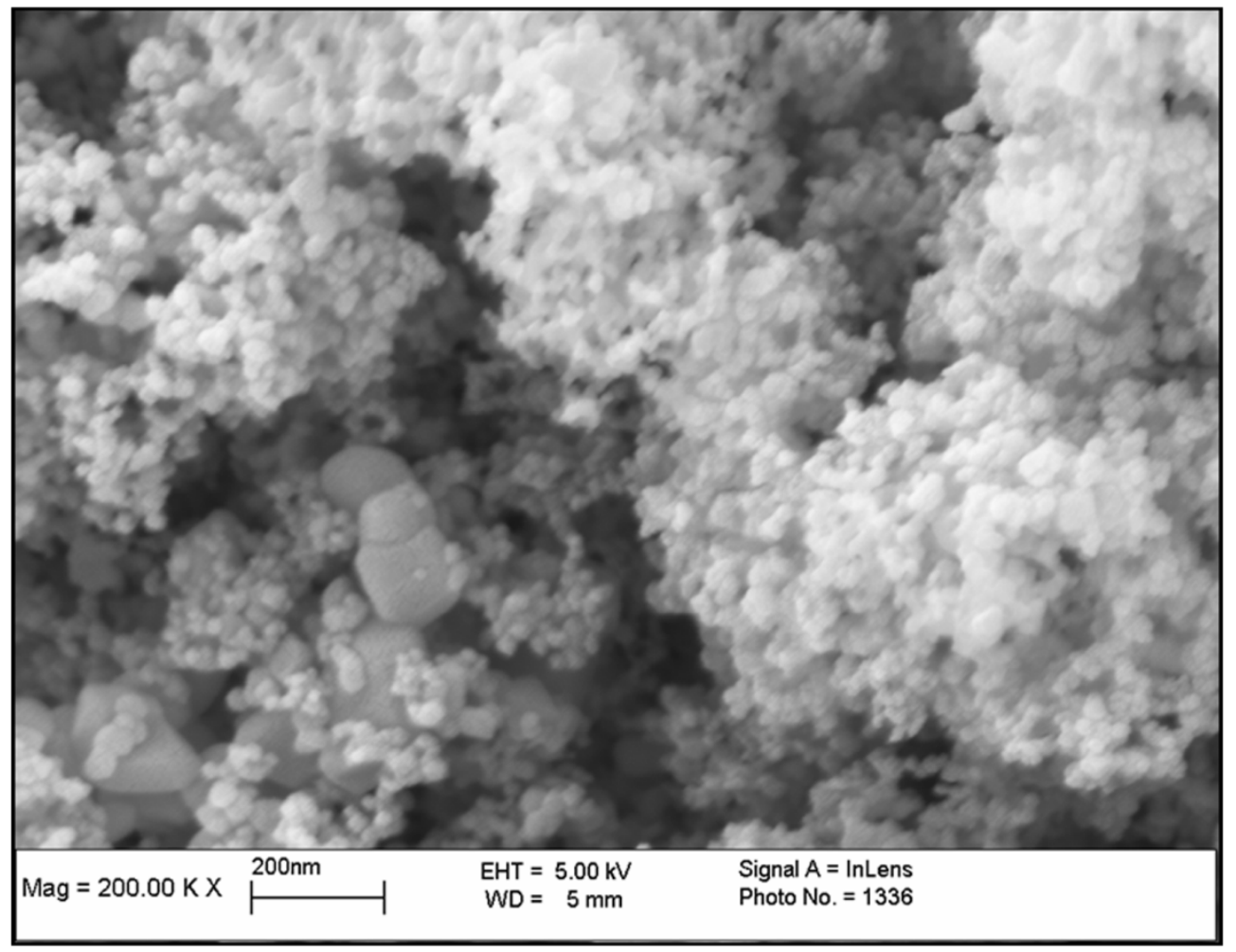

Figure 1 Scanning electron microscope image of P25 titanium dioxide 


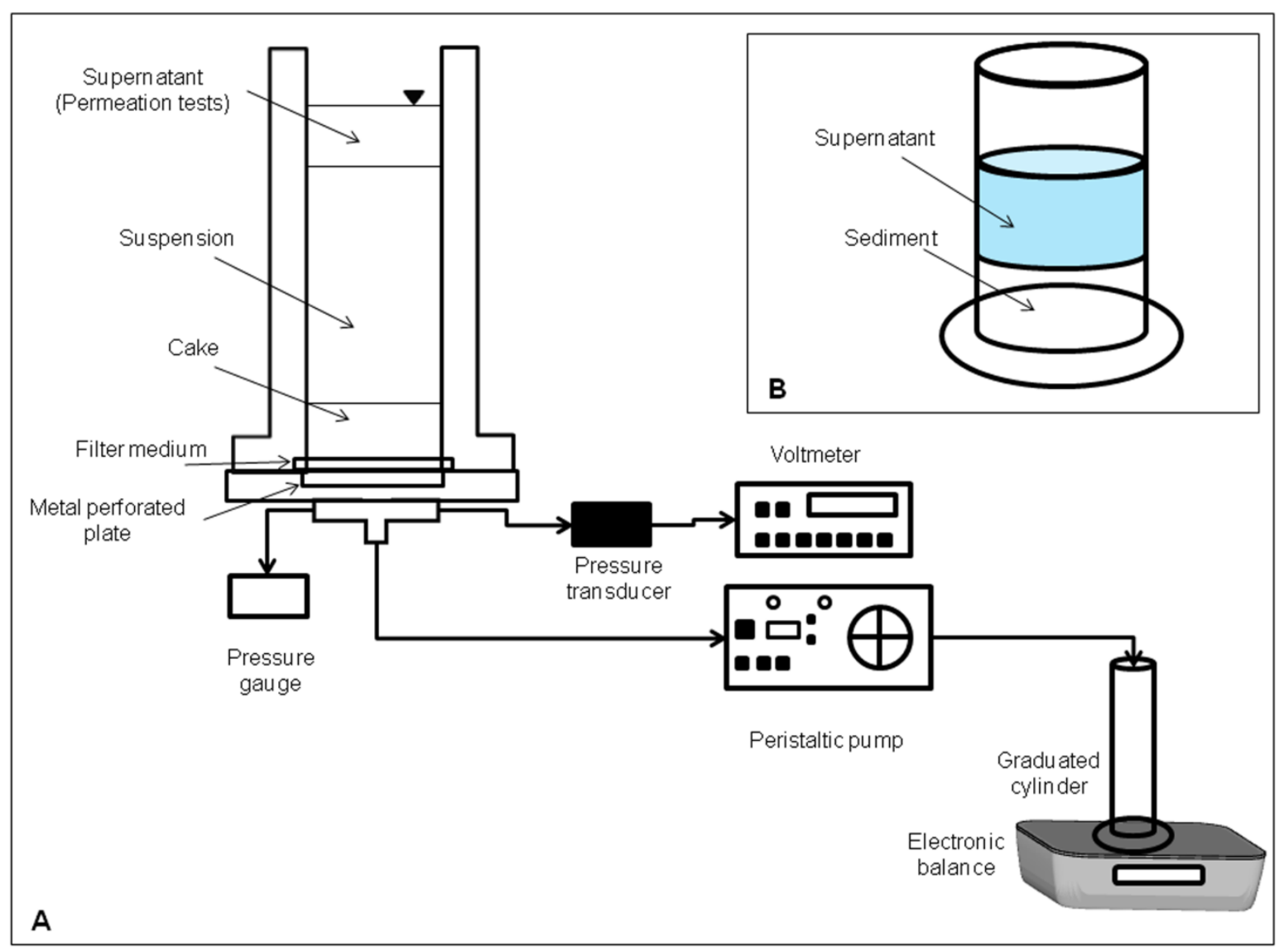

Figure 2 Experimental setup in filtration (A), permeation (A \& B) and sedimentation $(B)$ tests 


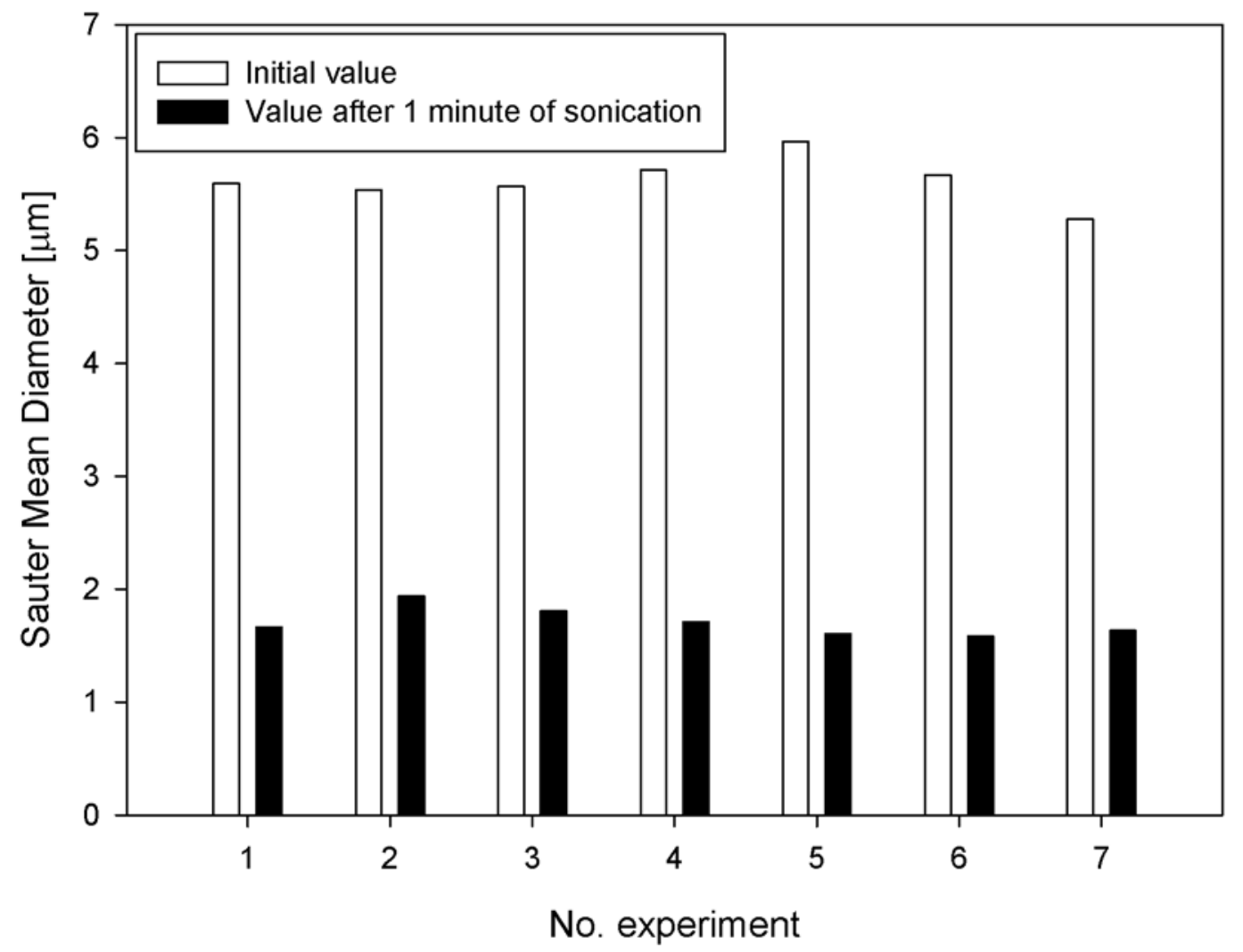

Figure 3 Sauter mean diameter of P25 clusters initially and after 1 minute of sonication at the lowest shear field under LD for 7 repeated experiments 


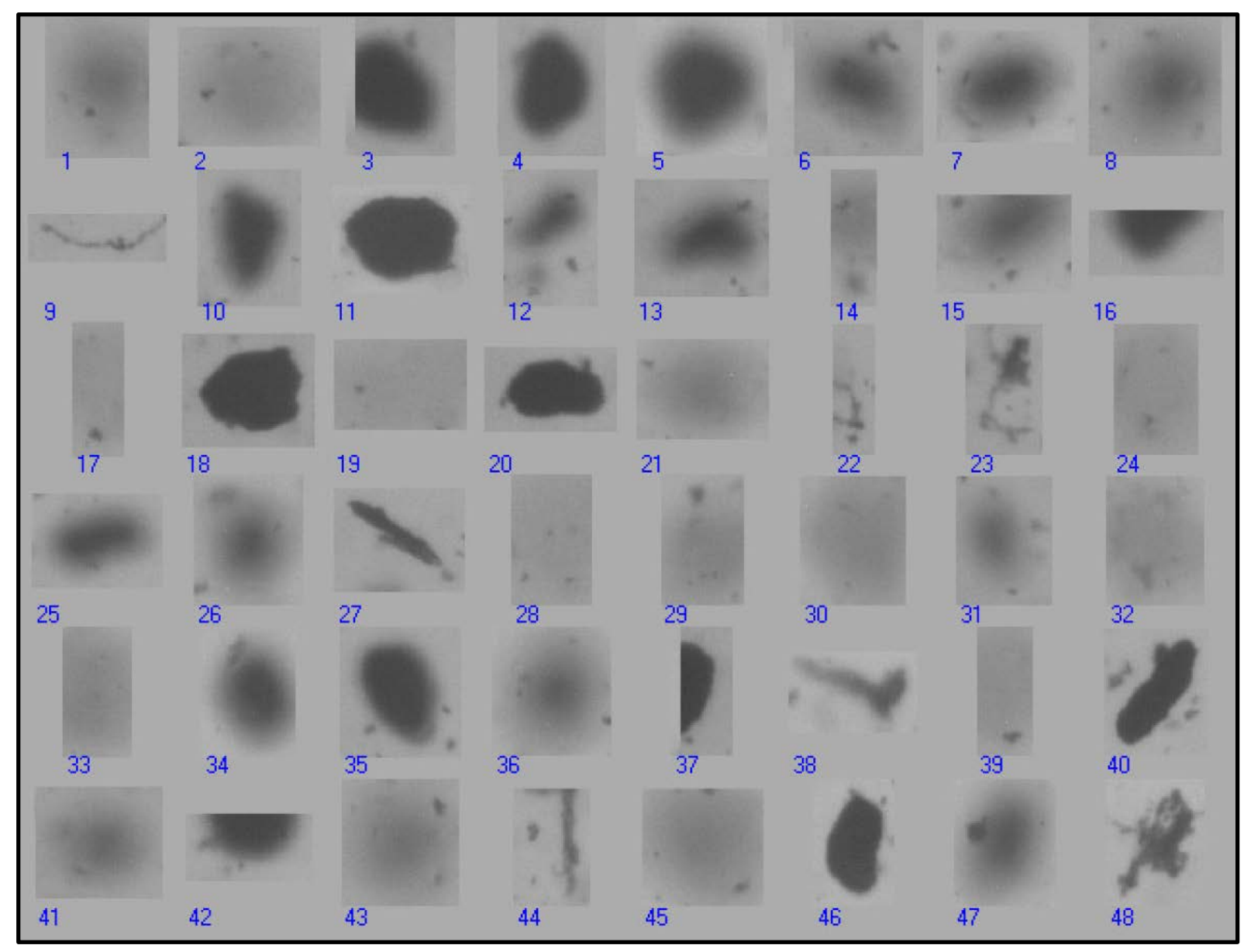

Figure 4 Cluster shapes determined by off-line image analysis: a sample of 48 of the images used 


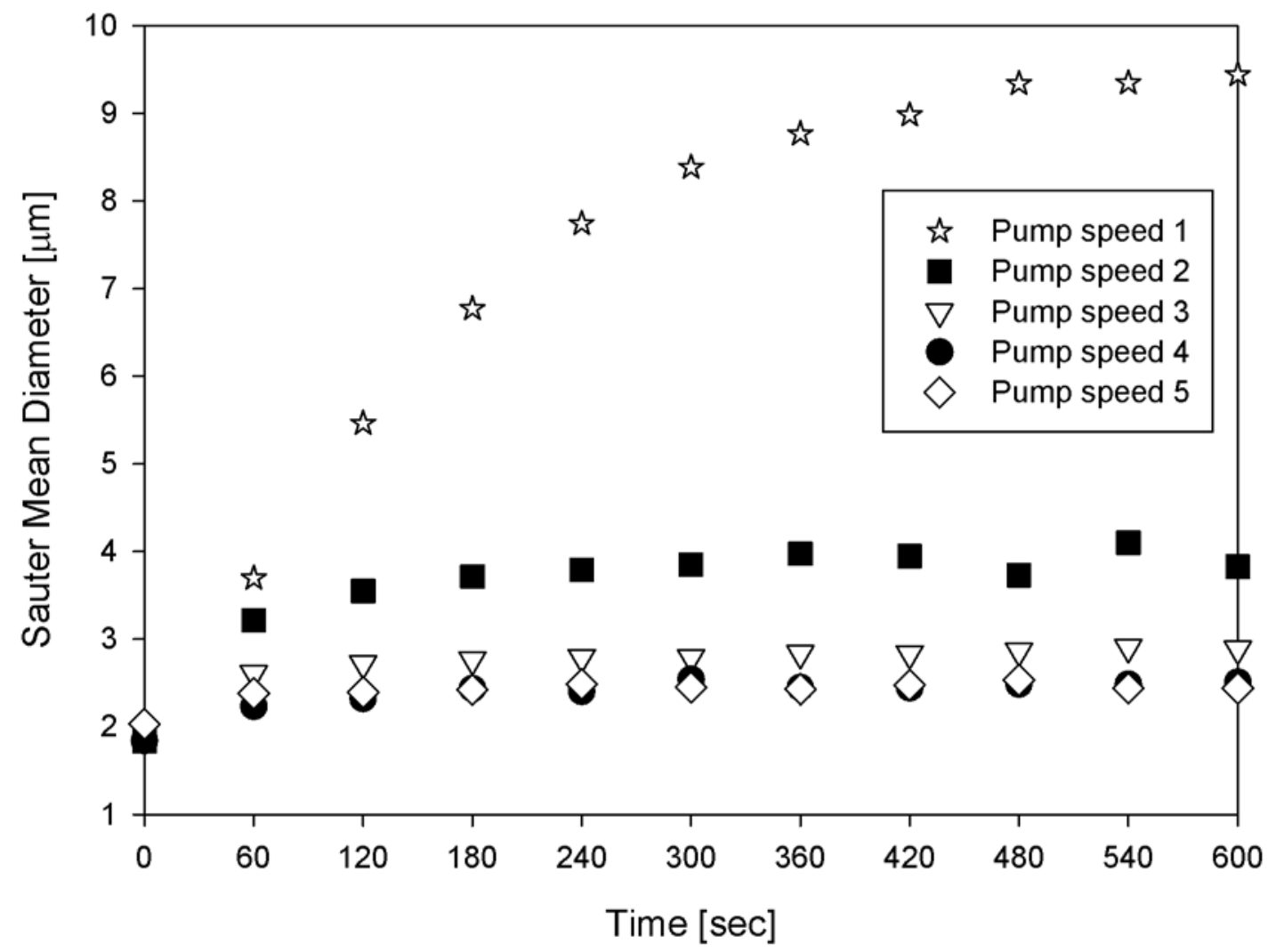

Figure 5 Variation of the Sauter Mean Diameter over time for five different values of shear fields created by increasing pump speed from 1 (lowest value) to 5 (highest) during the LD analysis 


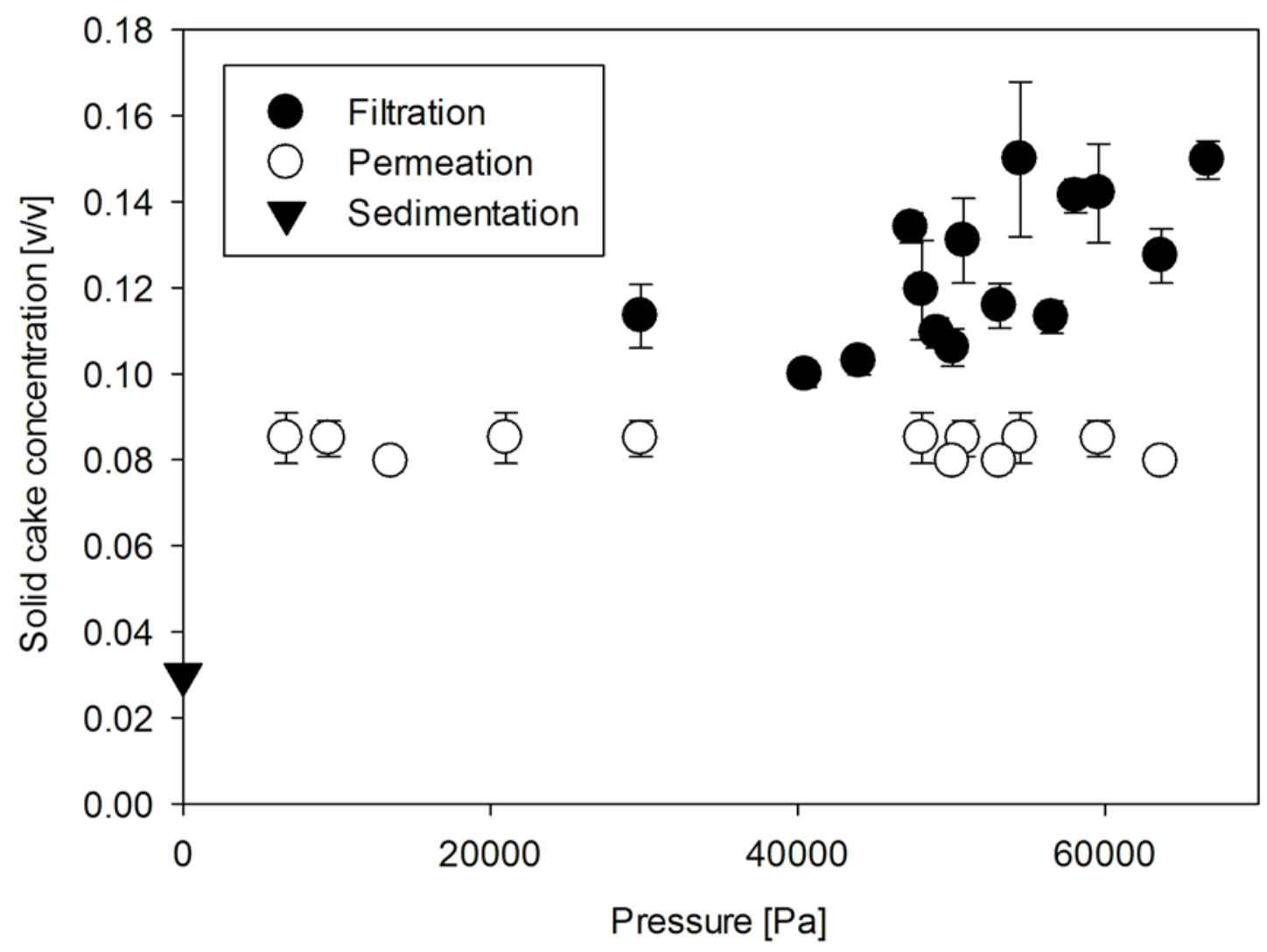

Figure 6 Dependence of solid cake concentrations on pressure in filtration, permeation and sedimentation processes 


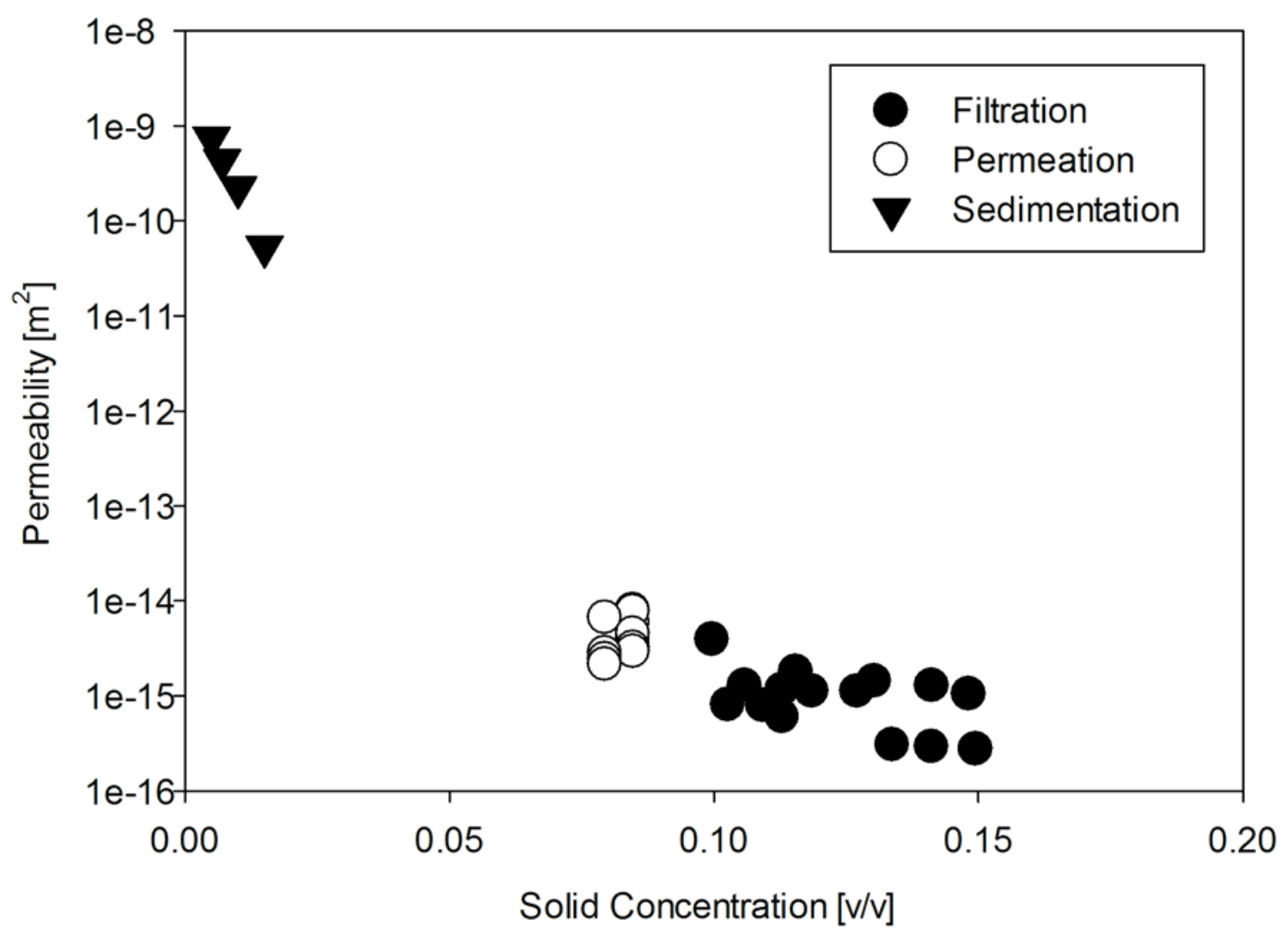

Figure 7 Deduced permeability with solid concentration in filtration, permeation and sedimentation tests 


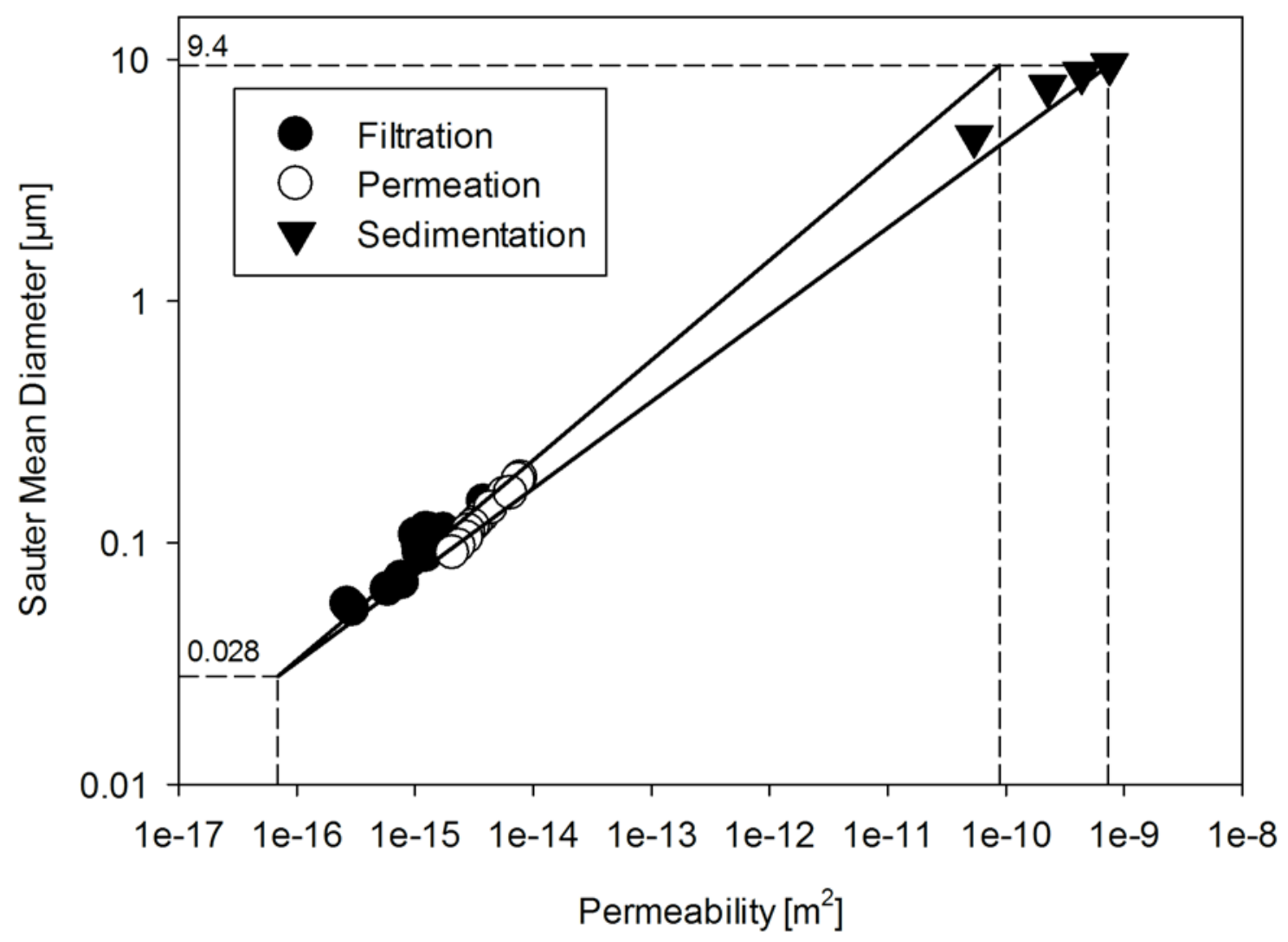

Figure 8 Sauter mean diameter and corresponding permeability deduced from experimental readings and the predicted operating envelope based on particle characterisation 